\title{
Paget-Schroetter Syndrome in the Young and Active
}

Viju Vijaysadan, MD, Aphrodite M. Zimmerman, MS, and Rafael E. Pajaro, MD

We report this case of effort thrombosis of the upper extremity (Paget-Schroetter syndrome) caused by hypertrophied muscles. This unusual cause of extrinsic venous compression and intimal injury leading to thrombosis was treated uniquely with good outcome. Untreated symptomatic patients can sustain long-term disability from venous obstruction resulting in significant loss of occupational productivity and quality of life. For the same reason, early catheter directed thrombolysis followed by anticoagulation and surgical intervention are recommended in much of the recent literature. Thrombolysis is the most common form of treatment, followed by surgery, if needed, after careful diagnostic approach. A majority of the literature supports a multimodal approach, but there is no definite consensus on management. This highlights the need for randomized clinical trials to guide management as well as to assess the safety and efficacy of anticoagulants commonly used and to define the optimal duration of therapy after thrombolysis. (J Am Board Fam Pract 2005;18:314-9.)

Paget-Schroetter syndrome (effort thrombosis of upper extremity) was described by Paget in 1875 and Schroetter in 1884 independently; Huges, while reviewing his cases of spontaneous venous thrombosis, gave it its name. Upper extremity deep vein thrombosis (UEDVT) refers to thrombosis of the axillary and/or subclavian veins. They are classified as primary and secondary based on pathogenesis. Primary UEDVT is a rare disorder (2 per 100,000 persons per year ${ }^{1}$ ), which comprises (1) Paget-Schroetter Syndrome, also known as effort thrombosis, and (2) idiopathic UEDVT. UEDVT has a potential for considerable morbidity because pulmonary embolism is present in up to a third of patients with UEDVT, ${ }^{2}$ and other complications, such as persistent pain and swelling, superior vena cava syndrome, and problems with vascular access, can be disabling. Secondary UEDVT develops in patients with upper extremity central venous catheters, ${ }^{3}$ pacemakers, or cancer and accounts for most cases of UEDVT.

Primary UEDVT refers either to effort thrombosis (also called Paget-Schroetter syndrome, or

Submitted, revised, 21 March 2005.

From the Department of Medicine, Overlook Hospital, Summit, New Jersey (VV, REP), and the Medical School, University of Medicine and Dentistry of New Jersey, Newark (AMZ).

Conflict of interest: none declared.

Corresponding author: Viju Vijaysadan, MD, Department of Medicine, Overlook Hospital, 99 Beauvoir Avenue, Summit, NJ 07901 (e-mail: viju@earthlink.net).
PSS) or idiopathic UEDVT. In contrast to patients with Paget-Schroetter syndrome, patients with idiopathic UEDVT have no known trigger or obvious underlying disease. Idiopathic UEDVT, however, may be associated with occult cancer. In one study, one fourth of patients presenting with idiopathic UEDVT were diagnosed with cancer (most commonly lung cancer or lymphoma) within 1 year of follow-up. Most of these cancers were discovered during the first week of hospital admission for the venous thrombosis. ${ }^{4}$ Young, healthy patients with PSS develop spontaneous UEDVT, usually in their dominant arm, after such strenuous activities such as rowing, wrestling, weight lifting, and baseball pitching. The heavy exertion causes microtrauma to the vascular intima and leads to activation of the coagulation cascade. Significant thrombosis may occur with repeated insults to the vein wall, especially if mechanical compression is also present. ${ }^{5}$ The optimal management of subclavian vein effort thrombosis remains a dilemma because outcomes after different treatment strategies are based on isolated case reports and small retrospective series. This decision should be based on the cause of thrombosis and should be individualized. ${ }^{6}$ We report a case of Paget-Schroetter syndrome caused by hypertrophied coracobrachialis and short head of the biceps in a very active young man.

\section{Case Reports}

The patient was a 19 year-old male who had been exercising in preparation to join the Marines. Two 
weeks before presentation, he experienced discomfort in the right antecubital fossa and felt a palpable firmness of the basilic and axillary veins. With continued physical activity, his arm began to swell progressively and became diffusely erythematous. He found that lifting his arm above the head relieved the swelling but caused paresthesias in his hand and fingers. This sensation resolved on lowering the arm.

The patient worked as a landscaper, and he used a weed-cutting machine for 8 to 9 hours a day. The patient denied any trauma or past injuries to his upper extremities or trunk. He denied chest pain, shortness of breath, fever, easy bruising, bleeding, palpitation, bone pain, lymph node or joint swelling. His past medical history was significant for a nondisplaced fracture of the tibia 7 months before this admission that had required a cast, which had been removed 4 months before the day of presentation. The patient was not on any medications, although a previous physician had prescribed a 7-day course of clarithromycin for a presumed right upper extremity cellulitis based on his presenting symptoms. The patient's family history was negative for any missed abortions, coagulopathies, venous thrombosis, or pulmonary embolism. The patient smoked half a pack of cigarettes per day and drank alcohol socially. He was an active member of his high school soccer, football, and wrestling teams.

On physical examination, the patient had prominent superficial veins visible over the right subclavian area and a palpable cord over the basilic and axillary veins. The right upper extremity had nonpitting edema with homogeneous erythema that blanched to palpation. His radial and brachial pulses were $2+$ at rest. He had a positive Wright test (attenuation of brachial/radial pulses with hyperabduction of the arm, caused by compression of the neurovascular structures in the subcoracoid region by the pectoralis tendon, the head of humerus, the coracoid process and muscles from coracoid process as short head of biceps and coracobrachialis), a negative Adson test (attenuation of radial pulse caused by narrowing of the space between scalenus anticus and medius, resulting in compression of the subclavian artery and the brachial plexus), and a negative Halsted test (attenuation of the radial pulse caused by narrowing the space between the first rib and the clavicle, thereby causing neurovascular compression) (Table 3). The extremities were compared, and there was no directly observed or relative atrophy of any group of muscles. Tinel and Phalen signs were absent, and no tenderness was noted over the supraclavicular fossa. The left upper extremity showed no abnormalities.

An upper extremity Doppler ultrasound showed complete thrombosis of the right subclavian axillary vein and its branches. Lower extremity Doppler ultrasound was negative for thrombus. A right upper extremity venogram confirmed occlusion of right subclavian, axillary, and upper brachial branches with thrombus. Computed tomography of the chest showed possible pulmonary embolism.

The patient was admitted for treatment with intravenous unfractionated heparin (weight-based protocol) and thrombolysis. Thrombolysis of the clot was performed using urokinase at 150,000 units/hour with a multisided hole infusion catheter positioned at the right basilic and cephalic veins. After 4 hours of thrombolysis, follow-up venogram showed partial lysis of clot in right axillary and basilic veins. Catheters were repositioned and thrombolysis was continued at 160,000 units/hour overnight. The next follow-up venogram showed residual clot in the subclavian vein despite 36 hours of transcatheter thrombolysis, and the patient was taken for mechanical thrombolysis with an Angiojet device. A postangioplasty venogram revealed the veins of right upper extremity to now be free of occlusion.

Echocardiographic and hematologic tests for hypercoagulable conditions were all within normal limits. A magnetic resonance imaging study of the

Table 1. Signs and Symptoms of Axillary-Subclavianvein Thrombosis versus Thoracic Outlet Syndrome

\begin{tabular}{lcc}
\hline Condition & Symptoms & Signs \\
\hline Axillary-subclavian thrombosis & $\begin{array}{c}\text { Vague shoulder or neck discomfort; arm, } \\
\text { hand edema }\end{array}$ & $\begin{array}{c}\text { Supraclavicular fullness, palpable cord, extremity } \\
\text { edema/cyanosis, jugular vein distention, } \\
\text { dilated veins, unable to access catheter } \\
\text { Thoracic outlet syndrome }\end{array}$ \\
& Pain radiating to arm; hand weakness & $\begin{array}{c}\text { Brachial plexus tenderness, arm/hand atrophy, } \\
\text { positive Adson or Wright test }\end{array}$ \\
\hline
\end{tabular}


Table 2. Imaging Modes Used and Their Risk and Benefits

\begin{tabular}{|c|c|c|}
\hline Diagnostic Test & Advantage & Disadvantage \\
\hline Duplex Ultrasound & Inexpensive, reproducible, noninvasive & $\begin{array}{l}\text { Failure to detect thrombus under clavicle in } \\
\text { subclavian vein }\end{array}$ \\
\hline CT & $\begin{array}{l}\text { Vessel compression from outside; may define } \\
\text { central thrombus }\end{array}$ & Contrast required; not validated in entirety \\
\hline MRI & $\begin{array}{l}\text { Detects central thrombus with accuracy; good } \\
\text { evaluation of collaterals }\end{array}$ & $\begin{array}{l}\text { Unsuitable for patients with metal implant or } \\
\text { pacemaker; claustrophobia }\end{array}$ \\
\hline
\end{tabular}

upper extremity was obtained to assess for sources of external venous compression. No masses or anomalous bony structures were identified along the course of the subclavian vein, however there was a relative hypertrophy of the coracobrachialis and short head of the biceps. The decision was made to anticoagulate the patient for 6 months with warfarin to achieve an international normalized ratio between 2 and 3. On discharge, the patient's symptoms were improved, and he was referred for a structured physical therapy program.

\section{Discussion}

Our patient developed a thrombus is the dominant arm after repeated strenuous activity involving hyperabduction at the shoulder. This heavy exertion along with the hypertrophied coracobrachialis and short head of the biceps caused microtrauma to the vessel intima and led to activation of coagulation cascade. Significant thrombosis can also occur if there is mechanical obstruction to the vessel wall. ${ }^{5}$
Compression of the subclavian vein typically occurs in young athletes with hypertrophied muscles who do weight lifting or completely abduct their arms. ${ }^{7}$

Although axillary and subclavian involvement can be asymptomatic, the patient can present with localized swelling to the arm and face or can even have associated visual or vestibular symptoms, depending on the site of obstruction. Our patient had localized swelling of the arm along with brachial plexus involvement, as evidenced by tingling sensation on hyper abduction. That a provocative test such as the Wright test was positive ${ }^{8}$ suggests compression of neurovascular structures in the subcoracoid region by musculoskeletal structures such as, in this case, the coracobrachialis and short head of the biceps.

Other clinical signs may be seen in UEDVT, such as low-grade fever that may be attributable to thrombosis and high-grade fever that may be suggestive of septic thrombophlebitis. Tachycardia may be present if there is a pulmonary embolism or

Table 3. Provocative Physical Tests

\begin{tabular}{|c|c|c|c|}
\hline Diagnostic Test & Maneuver & Positive Test & Mechanism \\
\hline Adson (scalene) & $\begin{array}{l}\text { The patient is instructed to } \\
\text { inspire maximally and hold his } \\
\text { or her breath while the neck } \\
\text { is fully extended and the head } \\
\text { is turned toward the affected } \\
\text { side. }\end{array}$ & $\begin{array}{l}\text { The loss or decrease of radial } \\
\text { pulse or the reproduction of } \\
\text { neurologic symptoms suggests } \\
\text { a positive test. }\end{array}$ & $\begin{array}{l}\text { The Adson (scalene) test causes } \\
\text { narrowing of the space } \\
\text { between the scalenus anticus } \\
\text { and medius, resulting in } \\
\text { compression of the subclavian } \\
\text { artery and the brachial plexus. }\end{array}$ \\
\hline Halsted (costoclavicular) & $\begin{array}{l}\text { The patient is instructed to } \\
\text { place his or her shoulders in a } \\
\text { military position (drawn } \\
\text { backward and downward). }\end{array}$ & $\begin{array}{l}\text { The loss or decrease of radial } \\
\text { pulse or the reproduction of } \\
\text { neurologic symptoms suggests } \\
\text { a positive test. }\end{array}$ & $\begin{array}{l}\text { The Halsted (costoclavicular) } \\
\text { test is used to narrow the } \\
\text { costoclavicular space between } \\
\text { the first rib and the clavicle, } \\
\text { thereby causing neurovascular } \\
\text { compression }\end{array}$ \\
\hline Wright (hyperabduction) & $\begin{array}{l}\text { To perform the test, the } \\
\text { patient's arm is hyperabducted } \\
180^{\circ} .\end{array}$ & $\begin{array}{l}\text { The loss or decrease of radial } \\
\text { pulse or the reproduction of } \\
\text { neurologic symptoms suggests } \\
\text { a positive test. }\end{array}$ & $\begin{array}{l}\text { The Wright (hyperabduction) } \\
\text { test causes the neurovascular } \\
\text { structures to be compressed in } \\
\text { the subcoracoid region by the } \\
\text { pectoralis tendon, the head of } \\
\text { the humerus, or the coracoid } \\
\text { process and muscles/tendons } \\
\text { arising from the process. }\end{array}$ \\
\hline
\end{tabular}




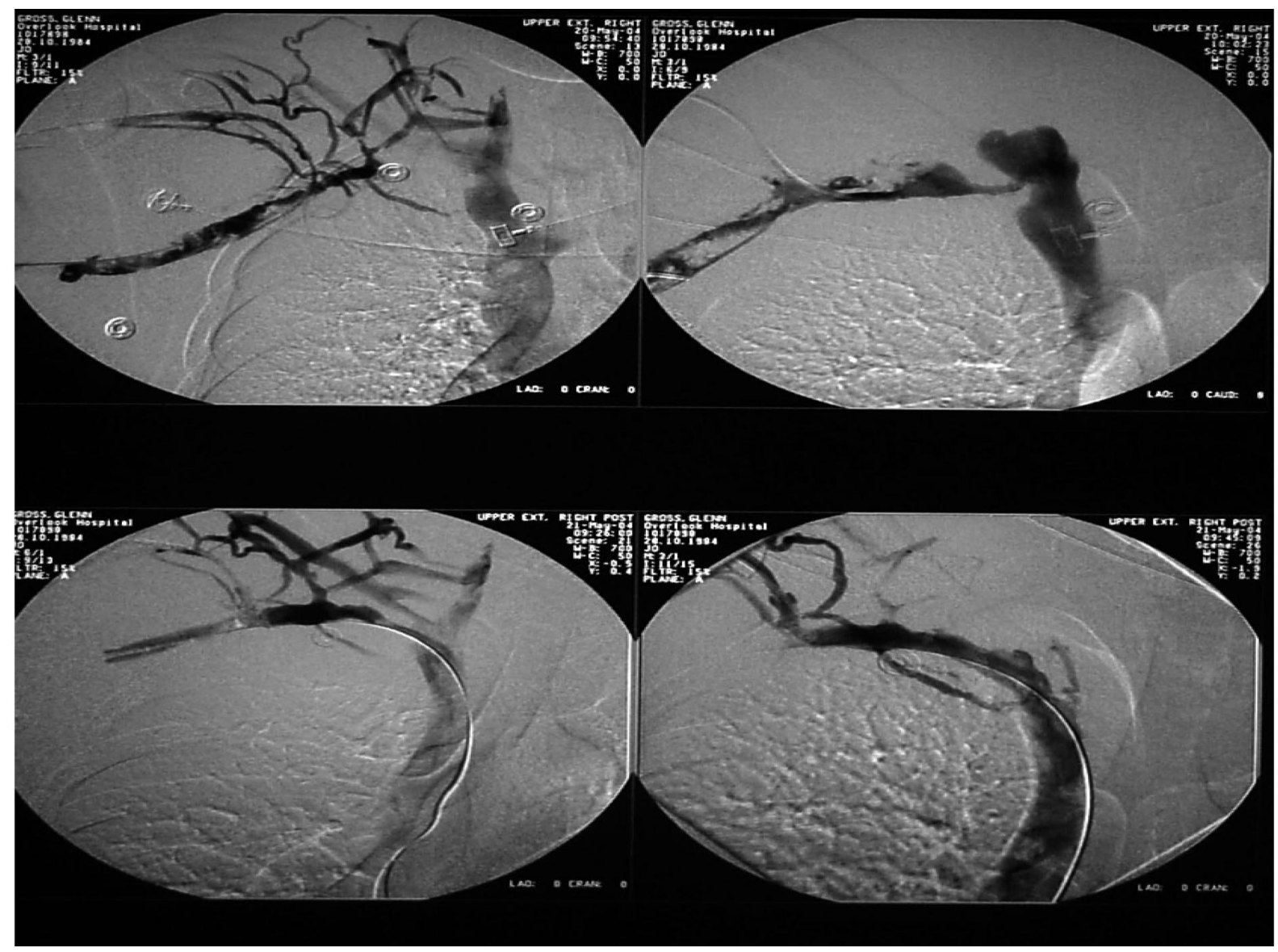

Figure 1. Venogram showing initial thrombus in the right brachial, axillary, and subclavian veins (upper left) and different stages of thrombolysis to successful lysis of thrombus (lower right).

superior vena cava obstruction. Physically robust people may have atypical presentations of PagetSchroetter Syndrome, such as pulmonary hypertension as a result of pulmonary embolism. ${ }^{9}$ Localized erythema, palpable tenderness, and paraesthesias are nonspecific and can occur in cellulitis, lymphedema, neoplastic compression of blood vessels, muscle injury, or superficial vein thrombosis, among other diagnoses (Table 1). Fewer than half of these symptomatic patients have imaging evidence of an UEDVT. ${ }^{2}$ Therefore, it is important to confirm or exclude the diagnosis with objective testing. ${ }^{2}$

Duplex Ultrasound is the initial imaging test of choice for diagnosis because it is noninvasive and demonstrates a high sensitivity and specificity for peripheral (jugular, distal subclavian, axillary) UEDVT. ${ }^{2}$ False-negative studies do occur, however, because of acoustic shadows from the clavicle, which limits complete visualization of the subclavian vein. ${ }^{10}$ Magnetic resonance imaging of the vessels and soft tissue is a very reliable noninvasive method for detecting thrombus and defining the regional anatomy. In the presence of thrombosis, magnetic resonance angiography has good correlation with contrast venogram when the latter is contraindicated. ${ }^{11}$

Contrast venogram is the standard diagnostic test for characterization of the anatomy but has several drawbacks-technical difficulty in cannulating an edematous arm and requirement of a contrast agent (especially in patients with impaired renal function) - and is contraindicated in pregnancy. Contrast venogram can be done in preparation for interventions such as catheter-directed thrombolytic infusion, mechanical instruments such as the Angiojet, angioplasty, and follow-up venograms to assess the response to the intervention.

Anticoagulation is the mainstay of the management of UEDVT. The goal is to prevent further propagation of thrombus and maintain patency of collateral veins. ${ }^{1}$ Many studies have validated the 
usage of low molecular weight unfractionated heparin and even direct thrombin inhibitor in deep vein thrombosis therapy as a bridge to warfarin therapy. ${ }^{12-14}$ The current standard is 6 months of oral anticoagulation. . $^{2,9,9,15}$

Young and active patients with Paget-Schroetter syndrome will have a significant risk for developing long-term consequences (have greater risk of losing higher function) and are usually treated more aggressively than the use of conventional anticoagulation alone. ${ }^{13,16,17}$ Thrombolysis restores venous patency early, minimizing damage to the vessel endothelium and reducing the risk of long-term complication; this is especially true of post-thrombotic syndrome, which is characterized by chronic arm and hand pain and swelling. ${ }^{12,13,16,18}$ In contrast, thrombolysis is rarely used for the treatment of lower extremity deep vein thrombosis because those patients are generally not sufficiently concerned by the potential risk of chronic leg swelling. The best thrombolysis candidates are young, otherwise healthy patients with primary UEDVT, patients with symptomatic superior vena cava syndrome, and those who require preservation of a mandatory central venous catheter.

Contraindications include active bleeding, neurosurgery within the preceding 2 months, a history of hemorrhagic stroke, hypersensitivity to the thrombolytic agent, and surgery within the preceding 10 days. The risks of systemic side effects from thrombolysis are minimized by the usage of catheter-directed thrombolysis. This strategy also has the advantage of achieving higher clot resolution rates. ${ }^{18}$ The best thrombolytic is urokinase ${ }^{18-20}$ as evidenced by its second comeback since its unavailability in 1999. Although there are no head-to-head controlled trials that compare thrombolytics, the general consensus is to use recombinant tissue plasminogen activator or urokinase.

Mechanical intervention with the Angiojet (Possis Medical, Inc., Minneapolis, MN), used in our patient, can evacuate a large quantity of thrombus and thereby limit the dose and duration of thrombolytic therapy. ${ }^{21}$ Repositioning of the catheter after a serial assay based on venography may be necessary to prevent collateral circulation from diverting the medication away from the thrombus. ${ }^{19}$ Good prognostic indicators for success with thrombolysis include the duration of the thrombus formation and time interval to the onset of therapy with thrombolytics (earlier treatment leads to bet- ter outcome). Most of the studies used thrombolytics in patients with a history of signs and symptoms a duration of up to 3 weeks if other variables coexist, such as age, cause of the condition, coexistent hematologic disorders, or malignancy. ${ }^{12,19,22}$ This was considered in our patient before the therapy in addition to other variables that would affect the success of the intervention. The best candidates tend to be the young and healthy, with primary UEDVT or Paget-Schroetter syndrome. ${ }^{12,19,22}$ To prevent clot or fibrin formation around the catheter during treatment with thrombolytics, heparin is usually given concurrently. ${ }^{12}$

If extrinsic venous compression is causing PSS, as in our patient, most vascular surgeons are in favor of early correction of anatomy, ${ }^{12,18,23}$ which may involve rib and/or clavicle resection, depending on the body parts involved. ${ }^{3}$ In our patient, intermittent compression was evident at the area of the hypertrophied coracobrachialis and short head of the biceps. This was confirmed by magnetic resonance imaging/magnetic resonance venography and venography done in neutral and shoulderabducted position. However, surgical intervention was deferred, because the obstruction was not due to skeletal abnormality.

Moreover, after thrombolysis, a trial of conservative therapy rather than aggressive early surgical decompression is the best option in any case of proven thoracic outlet syndrome. ${ }^{7,17}$ Hence, our patient was discharged with a structured physical therapy and weight loss program, which could obviate the need for surgery. Follow up at 3 and 6 months after hospitalization was uneventful, and the patient continues to do well, with no evidence of post-thrombotic syndrome.

In conclusion, a staged, multimodal approach to PSS can effectively restore venous patency, reduce the risk of rethrombosis, and return the patient to normal function. An effective strategy includes transcatheter thrombolytic therapy, at least 6 months of anticoagulation, and balloon angioplasty and surgical intervention if needed at a later stage. The lack of consensus of this potentially disabling condition highlights need for randomized clinical trials to guide management. Primary care physicians should be aware of this condition and its atypical presentations, because delayed recognition in a high-functioning persons can be potentially disabling. 


\section{References}

1. Lindbald B, Tengborn L, Bergqvist D. Deep vein thrombosis of the axillary-subclavian veins: epidemiologic data, effects of different types of treatment and late sequelae. Eur J Vasc Surg 1988;2:161-5.

2. Prandoni P, Polistena P, Bernardi E, et al. Upper Extremity deep vein thrombosis. Risk factors, diagnosis, and complications. Arch Intern Med 1997;157: 57-62.

3. Becker DM, Philbrick JT, Walker FB. Axillary and subclavian venous thrombosis. Prognosis and treatment. Arch Intern Med 1991;151:1934-43.

4. Girolami A, Prandoni P, Zanon E, Bagatella P, Girolami B. Venous thromboses of upper limbs are more frequently associated with occult cancer as compared with those of lower limbs. Blood Coagulation Fibrinolysis 1999;10:455-7.

5. Zell L, Kindermann W, Marschall F, et al. PagetSchroetter Syndrome in sports activites-case study and literature review. Angiology 2001;52:337-42.

6. Filis KA, Nguyen TQ, Olcott C 4th. Subclavian vein thrombosis caused by an unusual congenital clavicular anomaly in an atypical anatomic position. J Vasc Surg 2002;36:629-31.

7. Joffe HV, Goldhaber SZ. Upper-extremity deep vein thrombosis. Circulation 2002;106;1874-80.

8. Parziale JR, Akelman E, Weiss AP, Green A. Thoracic outlet syndrome. Am J Orthop 2002;29:35360.

9. Bliss S, Weinberger S, Meier M, Saint S. Clinical problem-solving. The unusual suspect. N Engl J Med 2002;347:1876-81.

10. Haire WD, Lynch TG, Lund GB, Lieberman RP, Edney JA. Limitations of magnetic resonance imaging and ultrasound-directed (duplex) scanning in the diagnosis of subclavian vein thrombosis. J Vasc Surg 1991;13:391-7.

11. Hartnell GG, Hughes LA, Finn JP, Longmaid HE 3rd. Magnetic resonance angiography of the central chest veins. A new gold standard? Chest 1995;107: 1053-7.

12. Hicken GJ, Ameli FM. Management of subclavian- axillary vein thrombosis: a review. Can J Surg 1998; 41:13-25.

13. Aburahma AF, Sadler DL, Robinson PA. Axillary subclavian vein thrombosis. Changing patterns of etiology, diagnostic, and therapeutic modalities. Am Surg 1991;57:101-7.

14. Savage KJ, Wells PS, Schulz V, et al. Outpatient use of low molecular weight heparin (Dalteparin) for the treatment of deep vein thrombosis of upper extremity. Thromb Haemost 1999;82:1008-10.

15. Adelman MA, Stone DH, Riles TS, Lamparello PJ, Giangola G, Rosen RJ. A multidisciplinary approach to the treatment of Paget-Schroetter syndrome. Ann Vasc Surg 1997;11:149-54.

16. Urchel HC, Razzuk MA. Paget-Schroetter syndrome: what is the best management? Ann Thorac Surg 2000;69:1663-9.

17. Khan SN, Stansby G. Current management of Paget-Schroetter syndrome in the UK. Ann R Coll Surg Engl 2004;86:29-34.

18. Machleder HI. Evaluation of a new treatment strategy for Paget-Schroetter syndrome: spontaneous thrombosis of the axillary-subclavian vein. J Vasc Surg 1993;17:305-15.

19. Fraschini G, Jadeja J, Lawson M, et al. Local infusion of urokinase for the lysis of thrombosis associated with permanent central venous catheter in cancer patients. J Clin Oncol 1987;5:672-8.

20. Seigel EL, Jew AC, Delcore R, Iliopoulos JI, Thomas JH. Thrombolytic therapy for catheter related thrombosis. Am J Surg 1993;166:716-9.

21. Kasirajan K, Gray B, Ouriel K. Percutaneous AngioJet Thrombectomy in the management of extensive deep venous thrombosis. J Vasc Interv Radiol 2001; 12:179-85.

22. Chang R, Horne MK 3rd, Mayo DJ, Doppman JL. Pulse-spray treatment of subclavian and jugular venous thrombi with recombinant tissue plasminogen activator. J Vasc Interv Radiol 1996;7:845-51.

23. Lee MC, Grassi CJ, Belkin M, Mannick JA, Whittemore AD, Donaldson MC. Early operative intervention after thrombolytic therapy for primary subclavian vein thrombosis: an effective treatment approach. J Vasc Surg 1998;27:1101-8. 